\title{
El laboratorio de investigación en la historia de la enfermería como productor de conocimiento y su contribucion a la historia de la enfermeria brasileña
} The research lab in the history of nursing (Laphe) as producer knowledge and its contribution to the history of Brazilian Nursing

O laboratória de pesquisa em história da enfermagem (Laphe) como produtor de conhecimento e sua contribuição para a história da enfermagem brasileira

\author{
Vanessa Costa Cavalcanti ${ }^{1}$ y Almerinda Moreira ${ }^{2}$
}

${ }^{1}$ Graduada em Enfermagem pela Escola de Enfermagem Alfredo Pinto/UNIRIO, aluna do curso de pós-graduação em nível de especialização, sob a forma de treinamento de serviço para enfermeiros, nos moldes de residência em enfermagem clínica cirúrgica na UNIRIO, integrante do Laboratório de Pesquisa em História da Enfermagem.

${ }^{2}$ Doutora em Enfermagem pela Universidade de São Paulo, Professora Associada e Diretora da Escola de Enfermagem Alfredo Pinto/UNIRIO, pesquisadora e membro fundador do Laboratório de Pesquisa em História da Enfermagem

Cómo citar este artículo en edición digital: Cavalcanti, V.C. y Moreira, A. (2015). El laboratorio de investigación en la historia de la enfermería como productor de conocimiento y su contribucion a la historia de la enfermaria brasileña.Cultura de los

Cuidados (Edición digital), 19, 41. Disponible en: http://dx.doi.org/10.14198/cuid.2015.41.11>

Correspondencia: grupo de Pesquisa no CNPq: Laboratório de Pesquisa de História da Enfermagem - LAPHE. Escola de Enfermagem Alfredo Pinto/UNIRIO. Universidade Federal do Estado do Rio de Janeiro.Rua Dr. Xavier Sigaud, 290 - Urca -

Rio de Janeiro - RJ - 22.290-180

Correo electrónico: enfavanessacavalcanti@gmail.com.

Recibido: 12/10//2014; Aceptado: 10/003/2015

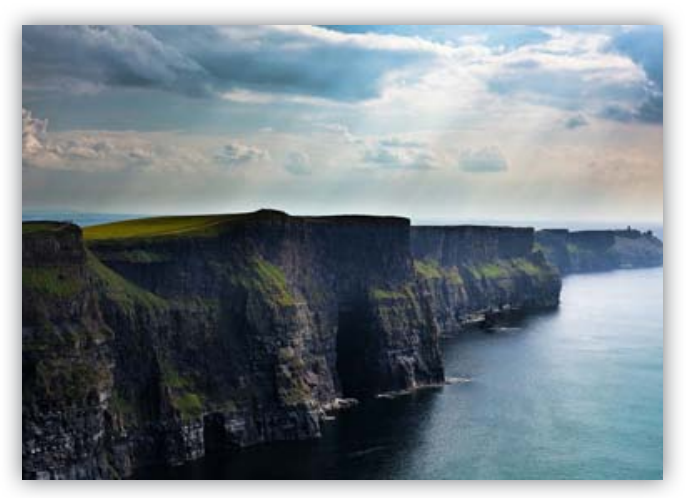

ABSTRACT

Study of historical nature that deals with the scientific production of 2008 to the first half of 2014 of the Laboratory for Research on the History of Nursing- Laphe. This reserch group was created on September 26, 2000, in the Alfredo Pinto Nursing School, Federal University of the State of Rio de Jeneiro and is registered at the National Center for Scientific and Technological Development. It was found that the group brings together researchers from the History of Nursing. According to the data analyzed in the period studied, there was a large academic production, thus contributing to the History of Nursing at both national and international level.

Keywords: History of Nursing, Laboratory of Resarch on the History of nursing, Scientific Production.

\section{RESUMEN}

Estudio de caracter historico que se ocupa de la produccion cientifica de 2008 hasta el primer semestre de 2014 de el Laboratorio de Investigacion sobre la Historia de la Enfermeria - Laphe. Este grupo de investigacion se creo 
en la Escuela de Enfermaria Alfredo Pinto, de la Universida Federal del Estado de Rio de Janeiro en 26 de septiembre de 2000, y es inscrita en el Centro Nacional de Desarrollo Cientifico y Tecnologico. Se encontro que el grupo esta formado por investigadores de la Historia de la Enfermaria. De acuerdo con los datos analizados en el periodo estudiado, hubo una gran produccion academica, contribuyendo asi a la Historia de la Enfermaria a nivel tanto nacional como internacional.

Palabras claves: Enfermaria, Historia de la Enfermaria, Laboratoria de Investigacion sobre la Historia de la Enfermaria, Produccion Cientifica

\section{RESUMO}

Estudo de natureza histórica que versa sobre a produção científica de 2008 ao primeiro semestre de 2014 do Laboratório de Pesquisa em História da Enfermagem - Laphe. Este grupo de Pesquisa foi criado em 26 de setembro de 2000, na Escola de Enfermagem Alfredo Pinto da Universidade Federal do Estado do Rio de Janeiro e é cadastrado no Centro Nacional de Desenvolvimento Científico e Tecnológico (CNPq). Constatou-se que o grupo congrega pesquisadores da História da Enfermagem. De acordo com os dados analisados no período estudado, observou-se uma grande produção acadêmica, contribuindo assim para a História da Enfermagem tanto a nível nacional quanto internacional.

Palavras-chave: Enfermagem, História da Enfermagem, Laboratório de Pesquisa em História da Enfermagem, Produção Científica.

\section{INTRODUÇÃO}

O Laboratório de Pesquisa de História da Enfermagem - LAPHE, da Escola de Enfermagem Alfredo Pinto - EEAP da Universidade
Federal do Estado do Rio de Janeiro - UNIRIO, criado no ano de 2000, e é um Grupo de Pesquisa cadastrado no Conselho Nacional de Desenvolvimento Científico e Tecnológico (CNPq). Tendo como líder do grupo o pesquisador Dr. Osnir Claudiano da Silva Junior, do Departamento de Enfermagem Fundamental, na época o único doutor do grupo. Com o imprescindível apoio dos professores Wellington Mendonça de Amorim do Departamento de Enfermagem de Saúde Pública, Fernando Porto do Departamento de Enfermagem Materno-Infantil e Almerinda Moreira do Departamento de Enfermagem Médico - Cirúrgica em conjunto fundaram em 26 de setembro de 2000, durante as comemorações dos 110 anos da Escola de Enfermagem Alfredo PintoEEAP, o referido laboratório.

Iniciado com apenas dois bolsistas de Iniciação Científica do Curso de Graduação em Enfermagem, hoje com reuniões semanais, conta com vários doutores, doutorandos, mestres, mestrandos, alunos de graduação da nossa escola, assim como, de várias outras renomadas instituições de ensino e pesquisa de nosso país, tais como: Escola de Enfermagem da Universidade de São Paulo - EEUSP, Escola de Enfermagem de Ribeirão Preto - EERP/ USP, Escola de Enfermagem da Universidade Estadual do Rio de Janeiro (UERJ), Escola de Enfermagem Anna Nery da Universidade Federal do Rio de Janeiro (UFRJ).

O grupo congrega pesquisadores e dedica-se ao desenvolvimento da Pesquisa em História da Enfermagem; além de buscar, preservar e recuperar documentos de interesse para a História da Enfermagem, facilitar o acesso ao acervo documental da EEAP e manter intercâmbio com pesquisadores e entidades ligadas à pesquisa em História da Enfermagem em níveis nacional e internacional. 
O principal objetivo do Laphe é o desenvolvimento da pesquisa e ensino para a História da Enfermagem no Brasil. Além disso, o grupo vem trabalhando nas frentes de pesquisa, ensino e extensão universitárias com pesquisadores em vários níveis de formação e experiências. Com o Arquivo Central da UNIRIO, desenvolvem-se ações de recuperação, conservação e disponibilização do acervo documental da Escola de Enfermagem Alfredo Pinto, aproximadamente 120 metros lineares de documentos textuais e cerca de 1000 fotografias.

Os estudos e pesquisas são sustentados basicamente pela exploração deste acervo. O produto das pesquisas subsidiam as disciplinas de História da Enfermagem na Graduação e pós-graduação em Enfermagem. A partir de 2003 realizam-se os Encontros dos Professores e Pesquisadores de História da Enfermagem e as Mostras da Produção Científica de História da Enfermagem do Rio de Janeiro, com a presença de pesquisadores e demais interessados na História da Enfermagem e da EEAP, em particular. A produção científica vem sendo divulgada em eventos nacionais e internacionais e publicações, preferencialmente indexadas e livros.

A estratégia laboratorial utilizada para a produção de conhecimento na área de História da Enfermagem pelo LAPHE, nos anos de 2008 a 2012, foi o objeto desta pesquisa.

\section{JUSTIFICATIVA}

A justificativa desta pesquisa é sustentada pelo conjunto de diversos trabalhos de pesquisa, fruto de projetos institucionais, teses de doutorado, dissertações de mestrado, trabalhos de final de curso como monografias de especialização e graduação em enfermagem e trabalhos de Iniciação Científica (IC) e ainda no Programa de Iniciação Científica do Conselho Nacional de Desenvolvimento Científico e Tecnológico (PIBIC/CNPq), desenvolvido por pesquisadores ligados ao Laboratório de Pesquisa de História da Enfermagem da Escola de Enfermagem Alfredo Pinto da UNIRIO e como esses trabalhos são ricos em informação. Com isso, foi desenvolvida essa pesquisa, visando fornecer um panorama da produção do Laphe durantes os seis anos e meio estudados, podendo com isso constatar a contribuição deste laboratório da EEAP com a História da Enfermagem.

\section{OBJETIVOS}

Para operacionalizar esse estudo foram traçados os seguintes objetivos:

- Caracterizar a produção intelectual do LAPHE.

- Discutir as contribuições das estratégias laborais na pesquisa de História da Enfermagem.

\section{METODOLOGIA}

Pesquisa exploratória documental de natureza histórica, baseada na análise da produção do LAPHE, cujo recorte temporal abrange período de 2008, quando o livro de atas apresenta-se mais detalhadamente descritos os projetos desenvolvidos no grupo, ao primeiro semestre do ano de 2014.

Segundo Silva Júnior (2011):

"A produção de um documento atende a uma necessidade administrativa, de uma organização, como um memorando, uma ordem de serviço, uma escala de atividades, o planejamento para um período de tempo, ou a necessidades individuais, como o planejamento de um dia numa agenda, uma lista de compras ou ainda suas necessidades afetivas como numa carta. 
Ou seja, estes registros foram produzidos com um fim específico e seguem seu destino. Contudo, uma vez preservados adquirem outro valor além da funcionalidade ou provas originais. Tornam-se documentos quando recolhidos num arquivo pessoal. $O$ acesso a estes documentos $e$ sua utilização para a pesquisa histórica altera o status dos registros para fontes históricas".

A reflexão histórica é parte relevante de uma construção intelectual e da consolidação do campo profissional e científico, conforme palavras do Professor Dr. Osnir Claudiano da Silva Junior, durante o IV Encontro de Professores e Pesquisadores de História da Enfermagem, promovido pelo LAPHE, em 2006, realizado durante a comemoração dos 116 anos da EEAP.

Segundo Moreira, Porto, Silva Junior e Amo$\operatorname{rim}(2008)$ :

"A história da enfermagem vem trilhando uma trajetória de persistência no conjunto de atividades consideradas fundamentais à formação do enfermeiro no Brasil. Ademais, reconhecemos a história da enfermagem como um instrumento de construção da memória coletiva da profissão, e um dos pilares importantes ao reconhecimento da permanência e das transformações da enfermagem na sociedade brasileira”.

\section{Locais de Pesquisa}

Os locais pesquisados foram a Biblioteca Setorial da Enfermagem do Centro de Ciências Biológicas e da Saúde da Universidade Federal do Estado do Rio de Janeiro, a Sala Professora Almerinda Moreira, local onde funciona o LAPHE, a secretaria dos Programas de Pós-graduação Mestrado em Enfermagem da UNIRIO - PPGENF e do Programa de Pós-Graduação Doutorado em Enfermagem e Biociências - PPGENBIO, o Núcleo de Pes- quisa de História da Enfermagem Brasileira - NUPHEBRAS da EEAN/UFRJ, o Laboratório de Estudos em História da Enfermagem-LAESHE da EERP/USP; Grupo de Pesquisa Historia e Legislação da Enfermagem EEUSP. Grupos esses onde foram desenvolvidos pesquisas pelos pesquisadores do LAPHE.

\section{Fontes de Pesquisa}

As fontes utilizadas no estudo foram as produções do Laboratório de Pesquisa de História da Enfermagem - LAPHE da EEAP, da UNIRIO, publicações do LAPHE, pesquisada nas bases de dados (Scielo, Lilacs, BVS, Index), livro de frequência e atas das reuniões.

Bibliografia de apoio foram as Revistas Científicas de Enfermagem, dissertações, teses e literatura que se aproxime do objeto de estudo.

A validação dos resultados ocorreu através da apresentação dos resultados parciais durante as reuniões semanais, com os pares de pesquisadores da História da Enfermagem, no Seminário Permanente, do Laboratório de Pesquisa de História da Enfermagem - LAPHE da EEAP, da UNIRIO; apresentação em eventos científicos e pareceres emitidos a partir da submissão aos periódicos científicos.

\section{Análise dos Dados}

Para a análise de dados, inicialmente os resumos foram submetidos a uma matriz de análise, que é um instrumento que facilita a visualização dos resultados da pesquisa, onde foram coletadas informações relativas ao: Nome do projeto, autor, ano que foi apresentado, publicação em periódicos e o orientador do projeto.

A pesquisa foi realizada de acordo com o Currículo Lattes, na base do CNPq, dos pesquisadores que constam nos livros de atas. 
Foram selecionados os Artigos Completos publicados em periódicos, os temas apresentados no Laphe e os temas de conclusão de curso, dissertações de mestrado e teses de doutorado, produzidos no grupo ou com participação dos pesquisadores e estudantes.

A participação em eventos também foi analisada de acordo com o Currículo Lattes dos pesquisadores, e com os Resumos Expandidos publicados em Anais de Eventos.

\section{RESULTADO E DISCUSSÃO DOS DADOS}

\section{Linhas e Projetos de Pesquisa}

Ao longo desses anos, foram desenvolvidas diversas pesquisas no Laphe, todas dentro da seguinte linha de pesquisa:

1. Desenvolvimento da Enfermagem Brasileira - que se propõe ao estudo e pesquisas sobre História da enfermagem, utilizando-se da interlocução com os conhecimentos das ciências humanas. Processo de profissionalização da enfermagem brasileira. Com estudos comparados sobre o processo de construção da enfermagem com os de outros países. Gênero na profissionalização da enfermagem, nas práticas e saberes das instituições de educação e saúde.

Porém os pesquisadores do Laphe criaram outros grupos e linhas, como as apresentada a seguir:

1. As políticas de saúde no contexto histórico social e suas implicações para a enfermagem de saúde pública no Brasil - Possibilitar por meio da produção do conhecimento e da interlocução com as ciências humanas e sociais uma discussão sobre as políticas de saúde e suas repercussões na trajetória da enfermagem de saúde pública no Brasil; Discutir os modos e as formas de produção e incorporação de tecnologias nas diversas configurações de enfermagem de saúde pública no Brasil; Analisar a partir das circunstâncias históricas e sociais a construção do saber da enfermagem de saúde pública no Brasil.

2. Bases Fundamentais, Culturais, Ambientais e Históricas do Cuidado - Concentra objetos de investigação de ordem macrossocial, destacada as questões sociais, ambientais e históricas. Centra-se em questões que envolvem políticas de saúde, ambiente e acultura dos sujeitos envolvidos no cuidado; trata o ambiente como elemento restaurador da saúde; discute o processo histórico da profissionalização do CUIDADO de enfermagem no Brasil e de estudos de História da Enfermagem e Biociências, utilizando-se da interlocução com conhecimentos das ciências humanas, sociais e biológicas, particularmente, com a História. Compara o processo de construção da Enfermagem Brasileira com as de outros países; discute os modos e as formas de produção e incorporação de tecnologias nas diversas configurações da Enfermagem no Brasil; Proporciona discussões sobre a profissionalização da Enfermagem nas práticas e saberes das instituições de educação e saúde.

Dentro dessas linhas de pesquisa, foram desenvolvidos os seguintes projetos de pesquisa:

1. De enfermeira a visitadora social - Pesquisa histórico-social sobre a especialização de enfermeiras através de curso na Escola de Enfermagem Alfredo Pinto 1927-1943. Situação: Concluído. 
2. Os primórdios da profissionalização da enfermagem no Brasil: formação e mercado de trabalho. 1888-1921 - Estudo histórico-social das mudanças ocorridas na enfermagem brasileira desde a libertação dos escravos, enquanto marco para ampliação do trabalho livre, e a reforma da primeira escola de enfermagem do Brasil em 1921. Os objetivos do estudo são: Identificar as circunstâncias de criação da EPEE e a estrutura da saúde no Estado brasileiro; analisar os elementos do processo de profissionalização da enfermagem, no período indicado e discutir o impacto da abertura da EPEE na profissionalização da enfermagem na capital da república. $\mathrm{O}$ conceito de profissão de $\mathrm{N}$ Elias e os estudos sobre o tema de Eliot Freidson são os pilares de interpretação dos dados. Os documentos do Arquivo Setorial da EEAP são as principais fontes da pesquisa. Situação: Concluído.

3. O acervo documental da Escola de Enfermagem Alfredo Pinto: 1890-1921 -Projeto de Pesquisa institucional. Situação: Concluído.

4. História do Cuidado nos aspectos micro e macromoleculares: prática, saberes e instituições - Estuda a trajetória do cuidado prestado ao ser humano por meio das práticas, saberes e instituições, considerando os aspectos sociais, culturais e históricos. Tem como objetivo geral: Investigar a trajetória do cuidado prestado ao ser humano por meio das práticas, saberes e instituições, considerando os aspectos sociais, culturais e históricos. O interesse para a realização deste projeto encontra-se na motivação pela necessidade de atender as deman- das da iniciação científica, da pós-graduação stricto senso mestrado e doutorado em enfermagem, objetivadas no âmbito da linha de pesquisa Bases Fundamentais, Culturais Ambientais e Históricas do Cuidado. Método e técnica: A pesquisa será embasada nas perspectivas da história social, micro história, história dos conceitos, nova história cultural, e dos estudos biográficos. As fontes a serem utilizadas no estudo são documentos escritos do tipo manuais, revistas, livros, relatórios, leis e decretos, manuscritos pessoais, iconografias e fac-símiles, utensílios e equipamentos utilizado na prática do cuidado. Tais fontes são consideradas com base fundamentais da História Nova divulgada nos estudos de Jacques Le Goff. As delimitações temporais, geográficas, documentais e objetais devem ser mais definidas nos subprojetos que integrarem esta investigação, considerando ato, fato e circunstâncias históricas. Situação: Em andamento.

5. Imagem pública da Enfermeira Brasileira, 1916-1931- Estudo histórico-social tendo por objeto de pesquisa o mecanismo de construção da imagem pública da enfermeira brasileira veiculada na imprensa ilustrada no distrito federal, delimitado nos anos 1916 e 1931. A metodologia conta com fontes primárias de documentos escritos e iconográficos, e as secundárias referentes aos estudos de aproximação com o objeto de estudo. A busca das fontes ocorrerá em bibliotecas e acervos situados no Rio de Janeiro e os resultados serão interpretados à luz das noções de Pierre Bourdieu. Situação: Em andamento. 
6. Os Dirigentes da Escola de Enfermagem Alfredo Pinto - Projeto desenvolvido com a colaboração de um Professor Mestre e dois bolsistas de Iniciação Científica/ UNIRIO, e está vinculado ao Laboratório de Pesquisa de História da Enfermagem LAPHE e a Linha de Pesquisa Desenvolvimento da Enfermagem Brasileira do Programa de Pós-graduação Mestrado em enfermagem da EEAP UNIRIO. Situação: Em andamento.

7. Registros noticiosos da Escola Profissional de Enfermeiros e Enfermeiras veiculados no Jornal do Commercio de 1890 a 1920 - Projeto trata da busca de noticias veiculadas no Jornal do Commercio, mídia escrita sobre a primeira escola de Enfermagem do país, a Escola Profissional de Enfermeiros e Enfermeiras do Hospício Nacional de Alienados, hoje Escola de Enfermagem Alfredo Pinto, com vistas a dar visibilidade e completar lacunas na historia da Escola e da Enfermagem brasileira. Situação: Concluído.

8. Influência das Políticas de Saúde na configuração da Enfermagem de Saúde Pública no Brasil no século XX: Demandas e Tendências - A pesquisa tem como objeto as configurações da enfermagem de saúde pública decorrentes das políticas de saúde no Brasil nos diversos contextos histórico-sociais do século XX. Objetivos Gerais: Investigar as circunstâncias apresentadas à enfermagem de saúde pública em sua luta simbólica para consolidar-se como saber e como prática, no Brasil, no transcorrer do século XX; e discutir os avanços e os limites impostos pelas políticas de educação e saúde para o desenvolvimento da enfermagem de saúde pública, no diversos contextos históricos e sociais, no Brasil. Trata-se de uma pesquisa de natureza histórico-social, para o desenvolvimento deste estudo será utilizada a análise documental. Situação: Concluído.

9. A reconfiguração da Escola de Enfermagem Alfredo Pinto no meado do século XX - Investiga os desdobramentos da reconfiguração do espaço social da Escola de Enfermagem Alfredo Pinto durante a gestão da primeira enfermeira na direção desta Escola (1943-1956). Trata-se de uma pesquisa de natureza histórico-social, para o desenvolvimento deste estudo será utilizada a análise documental. Adota-se o pensamento sociológico de Pierre Bourdieu, que nos respalda teoricamente para, não só, visualizar a reconfiguração da Escola de Enfermagem Alfredo Pinto como um espaço social, como também para analisar objetivamente o habitus e as relações de poder que se desenvolveram entre os agentes psiquiatras, as enfermeiras diplomadas e os alunos e alunas da Escola no campo da educação e saúde. Situação: Concluído.

Pelos dados expostos, pode se observar que dentre desses anos houve três linhas de pesquisa desenvolvidas. Nessas linhas houve nove projetos de pesquisa desenvolvidos no Laphe, sendo estes Trabalhos de Conclusão de Curso, Monografias dos cursos de especialização, Dissertação de Mestrado e Tese de Doutorado, os quais cinco estão concluídos, enquanto quatro prosseguem em andamento e a cada ano novas pesquisas são desenvolvidas. 


\section{Análise das publicações}

Pela matriz de análise pode-se perceber que no ano de 2010 dos trabalhos apresentados no Seminário Permanente do Laphe, houve um grande número de publicações em formato de artigo. Esse fato pode ser associada a comemoração dos 120 anos da Escola de Enfermagem Alfredo Pinto, onde aconteceu um maior estímulo a publicação, principalmente na Revista Online de Pesquisa Cuidado é Fundamental, que dedicou um número especial aos trabalhos do evento, como se observa no Gráfico 1.

Nos outros anos estudados, houve um maior número de trabalhos apresentados no Seminário Permanente do Laphe, porém os artigos completos publicados em periódicos não foram sua maioria. Deve-se estimular mais os pesquisadores a publicarem seus trabalhos, principalmente para revistas de outras instituições, e então aumentar mais a notoriedade do grupo.

\section{Disciplinas nos cursos de graduação e pós- -graduação}

Em relação ao ensino, há disciplinas obrigatórias e eletivas desenvolvidas pelos pesquisadores do Laphe, tanto no curso de graduação como na pós-graduação.

Mestrado e também como disciplinas optativas para o curso de graduação:

- Análise de fontes na pesquisa histórica pelos Professores Drs. Wellington Mendonça de Amorim, Fernando Porto, Almerinda Moreira e Professor Doutor Osnir Claudiano da Silva Jr.

- História e Profissionalização da Enfermagem no Brasil pelos Professores Drs. Wellington Mendonça de Amorim, Fernando Porto, Almerinda Moreira e Professor Doutor Osnir Claudiano da Silva Jr.
- Metodologia da Pesquisa pelo Professor Dr. Wellington Mendonça de Amorim.

Graduação:

- História da Enfermagem, disciplina obrigatória ministrada pelo Professor Dr. Osnir Claudiano da Silva Jr.

- Temas Emergentes em Enfermagem fundamental, disciplina eletiva ministrada pelos Professores Drs. Wellington Mendonça de Amorim, Fernando Porto, Almerinda Moreira e Professor Doutor Osnir Claudiano da Silva Jr.

- Identidade da Enfermagem em Saúde Pública no Brasil, disciplina eletiva, ministrada pelo Professor Dr. Wellington Mendonça de Amorim.

- História da Parturição, disciplina eletiva, ministrada pelo Professor Dr. Fernando Porto.

\section{Participação em eventos.}

A participação do grupo em eventos se faz através do incentivo à apresentação de trabalhos em congressos, seminários, jornadas.

No Quadro 1 tem detalhadamente os eventos que os pesquisadores do Laphe estiveram presente desde o ano de 2008 até o atual momento. Pode-se analisar que todos os anos há a participação na Jornada de Iniciação Científica da UNIRIO, comprovando que todo ano há bolsistas de iniciação científica acrescentando o produto de suas pesquisas ao grupo.

\section{Acervo Bibliográfico e Patrimônio}

O acervo bibliográfico do Laboratório de História da Enfermagem é constituído de banco de textos. Os livros específicos de História da Enfermagem estão disponíveis na Biblioteca Setorial da Enfermagem.

Seu patrimônio é composto da sala 502 da própria escola, além de computadores, mesas, televisão, estante e livros tudo adquirido através de projetos dos docentes além de diversas relíquias. 


\section{Intercâmbio entre Grupos}

O Laphe mantém estreito intercâmbio entre diversos grupos de pesquisa de História da Enfermagem como o Laboratório de Estudos em História da Enfermagem (LAESHE) da Escola de Enfermagem da USP em Ribeirão Preto, grupo que nasceu das reuniões do Laphe, Núcleo de Pesquisa de História da Enfermagem (NUPHEBRAS) da Escola de Enfermagem Anna Nery da Universidade Federal do Rio de Janeiro, Museu Nacional de Enfermagem Anna Nery (MUNEAN) em Salvador.

Não se pode esquecer de citar a Academia Brasileira de História da Enfermagem - ABRADHENF, com sede em São Paulo, foi fundada em 13 de Agosto de 2010, com o apoio integral dos pesquisadores do Laphe que fazem parte até hoje, como membros de sua diretoria. $\mathrm{Da}$ mesma forma aconteceu com a Federação Ibero Americana de História da Enfermagem, com sede na Espanha. Os pesquisadores do Laphe participaram ativamente de sua criação e são membros da diretoria. Assim sendo, constata-se que o grupo impulsiona a História da Enfermagem nos diversos níveis local, nacional e internacional.

Além disso, há grupos de pesquisa que nasceram partindo das reuniões do LAPHE, como o LACENF e LACUIDEM na própria EEAP. Esses grupos surgiram pela necessidade de aprofundamento de novas metodologias.

\section{CONCLUSÃO}

O Laphe foi criado há quatorze anos, porém o estudo é sobre os últimos seis de existência e produção acadêmica. Durante esse período, diversos profissionais puderam participar de suas reuniões semanais.

Permanece sendo um grupo de estudo da Escola de Enfermagem Alfredo Pinto que mantém as reuniões semanalmente, congregando alunos e pesquisadores de diversos níveis de formação acadêmica e instituição.

Os resultados do estudo demonstram que há alunos que começaram como discentes no Laphe entraram no Mestrado mantendo a linha de pesquisa e então passaram para o doutorado comprovando o crescimento profissional e pessoal que o grupo desenvolve.

O número de artigos publicados a partir de pesquisas no Laboratório de Pesquisa em História da Enfermagem é notável. Sua maioria foi publicada na Revista de Pesquisa Cuidado é Fundamental, revista online da Escola de Enfermagem Alfredo Pinto. Conclui-se que o grupo necessita investir em publicações para outras revistas, para então aumentar sua notoriedade.

Pela análise dos dados, pode ser percebido que a contribuição do Laphe para a História da Enfermagem é notável. Muitos professores estiveram presentes nas reuniões contribuindo para o crescimento profissional do grupo. Assim como, grandes nomes da História da Enfermagem passaram pelo grupo.

Conclui-se que o Laboratório contribuiu para a História da Enfermagem, gerando novos grupos de estudos, participando dos diversos eventos na área, incentivando a pesquisa, publicando diversos artigos, incrementando o crescimento profissional dos que o frequentam. Além de ampliar o conhecimento produzido para além das fronteiras do Brasil.

\section{REFERÊNCIAS}

- Geovannini, T., Moreira, A., Schoeller, D. y Machado, W. (2010). História da Enfermagem: versões e interpretações. Rio de Janeiro, Revinter.

- Moreira, A. y Oguisso, T. (2005). Profissionalização da Enfermagem Brasileira. Rio de Janeiro: Guanabara Koogan.5. 
- Moreira, A., Porto, F., Silva Junior, O.C., Amorim.W. (2008). Laboratório de Pesquisa em História da Enfermagem - LAPHE: História, Memória \& Relíquias..

- Oliveira, I.C.S., Lourenço, L.H.S.C. y Santos, T.C.F. (1997). Núcleo de Pesquisa de História da Enfermagem Brasileira (NUPHEBRAS). Escola Anna Nery, Revista de Enfermagem.
- Silva Junior, O.C. (2006). ANAIS do IV Encontro de Professores e Pesquisadores de História da Enfermagem e IV Mostra da Produção Científica de História da Enfermagem no Rio de Janeiro Resumos.

- Souza, G.F., Amorim, W. y Lopes, G.T. (2010). A Produção Científica em História da Enfermagem nas Jornadas de Iniciação Científica da UNIRIO (2002-2009). Revista de Pesquisa Cuidado é Fundamental, 2(3),1017-1028.

\begin{tabular}{|c|c|c|c|c|c|}
\hline 2013 & 2012 & 2011 & 2010 & 2009 & 2008 \\
\hline \multirow[t]{2}{*}{$\begin{array}{l}65^{\circ} \text { Congresso } \\
\text { Brasileiro de } \\
\text { Enfermagem }\end{array}$} & $\begin{array}{l}3^{\circ} \text { Colóquio } \\
\text { Latino } \\
\text { Americano de } \\
\text { História da } \\
\text { Enfermagem }\end{array}$ & $\begin{array}{c}10^{\circ} \text { Encontro } \\
\text { de Pesquisa } \\
\text { em Educação } \\
\text { da Região } \\
\text { Sudeste- Pós } \\
\text { graduação em } \\
\text { Educação }\end{array}$ & $\begin{array}{l}12^{\circ} \text { Seminário } \\
\text { Nacional de } \\
\text { Diretrizes para } \\
\text { Educação em } \\
\text { Enfermagem }\end{array}$ & $\begin{array}{l}\text { II Congresso } \\
\text { Cientiarum } \\
\text { Historia }\end{array}$ & $\begin{array}{l}11^{2} \text { Jornada } \\
\text { Nacional de } \\
\text { História da } \\
\text { Enfermagem }\end{array}$ \\
\hline & $\begin{array}{c}\text { Simpósio } \\
\text { Nacional: o } \\
\text { Cuidar em } \\
\text { Saúde e } \\
\text { Enfermagem }\end{array}$ & $\begin{array}{l}1^{\circ} \text { Seminário } \\
\text { de Educação e } \\
\text { Pesquisa em } \\
\text { História da } \\
\text { Enfermagem }\end{array}$ & $\begin{array}{l}\text { II Encontro de } \\
\text { História da } \\
\text { Educação do } \\
\text { Estado do Rio } \\
\text { de Janeiro }\end{array}$ & $\begin{array}{l}15^{\circ} \text { Seminário } \\
\text { Nacional de } \\
\text { Pesquisa em } \\
\text { Enfermagem }\end{array}$ & $\begin{array}{c}7^{\mathrm{a}} \text { Jornada de } \\
\text { Iniciação } \\
\text { Científica da } \\
\text { Unirio }\end{array}$ \\
\hline \multirow[t]{5}{*}{$\begin{array}{l}12^{\mathrm{a}} \text { Jornada de } \\
\text { Iniciação } \\
\text { Cientifica da } \\
\text { UNIRIO }\end{array}$} & $\begin{array}{l}10^{\circ} \text { Congresso } \\
\text { Internacional } \\
\text { da Rede Unida }\end{array}$ & $\begin{array}{c}14^{\mathrm{a}} \text { Jornada } \\
\text { Nacional de } \\
\text { História da } \\
\text { Enfermagem }\end{array}$ & $\begin{array}{l}9^{a} \text { Jornanda de } \\
\text { Iniciação } \\
\text { Científica da } \\
\text { UNIRIO }\end{array}$ & $\begin{array}{l}\text { VI Semana } \\
\text { Professora } \\
\text { Glete de } \\
\text { Alcântara }\end{array}$ & $\begin{array}{c}\text { V Semana } \\
\text { Professora } \\
\text { Glete de } \\
\text { Alcântara }\end{array}$ \\
\hline & $\begin{array}{l}64^{\circ} \text { Congresso } \\
\text { Brasileiro de } \\
\text { Enfermagem }\end{array}$ & $\begin{array}{l}10^{\mathrm{a}} \text { Jornada de } \\
\text { Iniciação } \\
\text { Cientifica da } \\
\text { UNIRIO }\end{array}$ & $\begin{array}{l}120 \text { Anos da } \\
\text { Escola de } \\
\text { Enfermagem } \\
\text { Alfredo Pinto: } \\
\text { História, } \\
\text { formação, } \\
\text { tecnologia e } \\
\text { inovação na } \\
\text { prática do } \\
\text { cuidado de } \\
\text { enfermagem }\end{array}$ & $\begin{array}{c}\text { III Mostra } \\
\text { Cientifica de } \\
\text { História da } \\
\text { Enfermagem }\end{array}$ & $\begin{array}{c}\text { II Mostra } \\
\text { Cientifca de } \\
\text { História da } \\
\text { Enfermagem }\end{array}$ \\
\hline & $\begin{array}{c}3^{a} \text { Semana de } \\
\text { Enfermagem } \\
\text { da } \\
\text { Universidade } \\
\text { Federal de } \\
\text { Viçosa }\end{array}$ & \multirow[t]{3}{*}{$\begin{array}{c}63^{\circ} \text { Congresso } \\
\text { Brasileiro de } \\
\text { Enfermagem }\end{array}$} & \multirow[t]{3}{*}{$\begin{array}{l}62^{\circ} \text { Congresso } \\
\text { Brasileiro de } \\
\text { Enfermagem }\end{array}$} & $\begin{array}{l}\text { VI Seminário } \\
\text { Nacional do } \\
\text { Centro de } \\
\text { Memória }\end{array}$ & $\begin{array}{c}\text { VI Encontro } \\
\text { de Professores } \\
\text { e VI Mostra de } \\
\text { Produção } \\
\text { Científica de } \\
\text { História da } \\
\text { Enfermagem }\end{array}$ \\
\hline & \multirow[t]{2}{*}{$\begin{array}{c}15^{\mathrm{a}} \text { Jornada } \\
\text { Nacional de } \\
\text { História da } \\
\text { Enfermagem }\end{array}$} & & & $\begin{array}{l}\text { Encontro } \\
\text { Luso- } \\
\text { Brasileiro de } \\
\text { História da } \\
\text { Ciência }\end{array}$ & $\begin{array}{l}\text { I Colóquio de } \\
\text { História da } \\
\text { Enfermagem }\end{array}$ \\
\hline & & & & $\begin{array}{l}8^{\mathrm{a}} \text { Jornada de } \\
\text { Iniciação } \\
\text { Científica da } \\
\text { UNIRIO }\end{array}$ & $\begin{array}{c}60^{\circ} \text { Congresso } \\
\text { Brasileiro de } \\
\text { Enfermagem }\end{array}$ \\
\hline
\end{tabular}

Quadro 1. Participação do Laphe em eventos do ano de 2008 ao primeiro semestre de 2014. 